\title{
SHAPE EQUIVALENCE DOES NOT IMPLY CE EQUIVALENCE1
}

\author{
STEVE FERRY
}

\begin{abstract}
We give an example of shape equivalent compacta $X$ and $Y$ such that
\end{abstract} there is no compactum $Z$ with cell-like maps $Z \rightarrow X$ and $Z \rightarrow Y$.

A space $X$ is said to be cell-like if for some imbedding of $X$ in an ANR, $X$ has the property that for each neighborhood $U$ of $X, X$ contracts to a point in $U$. This is an intrinsic property of $X$ and is independent of the choice of ANR and embedding. A continuous map $f: Z \rightarrow Y$ between compacta is said to be cell-like (CE) if $f$ is surjective and $f^{-1}(y)$ is cell-like for each $y \in Y$. If $X$ and $Y$ are compacta, we say that $X$ and $Y$ are $C E$ equivalent if there exist compacta $X=X_{0}, X_{1}, \ldots, X_{2 k}=Y$ and CE maps $f_{2 i}: X_{2 i+1} \rightarrow X_{2 i}$ and $f_{2 i+1}: X_{2 i+1} \rightarrow X_{2 i+2}$ for $i=0,1, \ldots, k-1$.

In $\left[\mathbf{F}_{1}\right]$ it is shown that two compacta which are homotopy equivalent must be $\mathrm{CE}$ equivalent. In fact, more is shown. The maps constructed have sections and contractible point-inverses. It is natural to seek a Čech analog of this theorem for general compacta. Thus, we are led to study the question: "If $X$ and $Y$ are shape equivalent compacta, must $X$ and $Y$ be CE equivalent?"

In this note we will exhibit a simple example which shows that this is not the case. Let $X$ be a plane compactum which is the union of a circle $C$ and a ray $R$

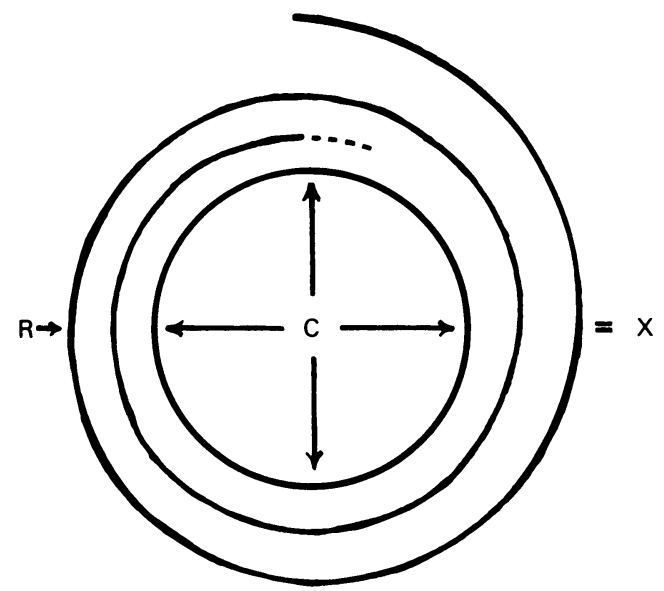

FIGURE 1

Received by the editors November 13, 1978 and, in revised form, April 29, 1979.

AMS (MOS) subject classifications (1970). Primary 57C10; Secondary 54C10, 55B05

Key words and phrases. Shape theory, cell-like map.

${ }^{1}$ Research partially supported by NSF grants and the A. P. Sloan Foundation. 
which spirals into $C$. See Figure 1. $X$ is shape equivalent to $S^{1}$. We will show that $X$ is not CE equivalent to $S^{1}$.

DEFINITION 1. We will say that a compactum $Z$ is an acyclic image if there exist a compactum $W$ with $\check{H}^{*}(W)=\check{H}^{*}(\mathrm{pt})$ and a continuous surjection $f: W \rightarrow Z$.

Lemma 1. Let $P$ and $Q$ be $C E$ equivalent compacta. Then $P$ is an acyclic image if and only if $Q$ is an acyclic image.

Proof. It suffices to consider the case in which there is a CE map $r: P \rightarrow Q$. It is clear that $Q$ is an acyclic image if $P$ is an acyclic image. Suppose, then, that $Q$ is an acyclic image. Let $f: W \rightarrow Q$ be a surjection as in Definition 1 and let $E$ be the pullback in the diagram below.

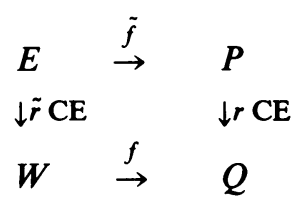

$E$ is compact, $\tilde{f}$ is surjective, and $\tilde{r}$ is CE. A cell-like set has the Čech cohomology of a point, so the Vietoris-Begle theorem $[\mathbf{S}]$ implies that $\tilde{r}$ induces an isomorphism of Čech cohomology. Thus, $E$ has the Čech cohomology of a point and $P$ is an acyclic image.

LEMMA $2^{2}$ The space $X$ of Figure 1 is not an acyclic image.

Proof. Suppose not. Let $f: W \rightarrow X$ be a surjection as in Definition 1. Let $r$ : $X \rightarrow C$ be a radial retraction and let $e: E^{1} \rightarrow C$ be the universal cover. Since $\check{H}^{1}(W) \cong[W, C]=0$, the composition $r \circ f: W \rightarrow C$ lifts to $E^{1}$ and there is a map $\tilde{f}: W \rightarrow E^{1}$ such that $e \circ \tilde{f}=r \circ f$.

Let $W^{\prime}=f^{-1}(R)$. Choose a map $\tilde{r}: R \rightarrow E^{1}$ so that $e \circ \tilde{r}=r \mid R$ and so that $\tilde{r} \circ f=\tilde{f}$ for some point $w_{0} \in W^{\prime}$. Let $W^{\prime \prime}=\left\{w \in W^{\prime} \mid \tilde{r} \circ f(w)=\tilde{f}(w)\right\}$. The usual argument shows that $W^{\prime \prime}$ is open in $W^{\prime}$ and therefore in $W . W^{\prime \prime}$ cannot be closed in $W$ since $W$ is connected and $W^{\prime \prime}$ is neither empty nor all of $W$.

There is therefore a sequence $\left\{w_{i}\right\} \in W^{\prime \prime}$ converging to a point $w^{*} \in W-W^{\prime}$. Thus, $\lim f\left(w_{i}\right) \in C$ and $\left\{\tilde{r} \circ f\left(w_{i}\right)\right\}$ is unbounded in $E^{1}$. On the other hand, $\left\{\tilde{r} \circ f\left(w_{i}\right)\right\}=\left\{\tilde{f}\left(w_{i}\right)\right\} \subset \tilde{f}(W)$, which is compact. This is the desired contradiction.

This completes the proof of our main result, since there is a continuous map of $[0,1]$ onto $S^{1}$.

\footnotetext{
${ }^{2}$ Lemma 2 is essentially Theorem 1 of M. K. Fort [FO].

It would be interesting to find shape equivalent $U V^{1}$ compacta which are not $\mathrm{CE}$ equivalent. Parts of $\left[F_{2}\right]$ are relevant to this problem.
} 


\section{REFERENCES}

[F $\mathbf{F}_{1}$ Steve Ferry, Homotopy, simple homotopy, and compacta, Topology (to appear).

$\left[\mathrm{F}_{2}\right] \ldots$ A stable converse to the Vietoris-Smale theorem with applications to shape theory, Trans. Amer. Math. Soc. (to appear).

[F0] M. K. Fort, Jr., Images of plane continua, Amer. J. Math. 81 (1959), 541-545.

[S] E. H. Spanier, Algebraic topology, McGraw-Hill, New York, 1966.

School of Mathematics, Institute for AdVanced Study, Princeton, New Jersey 08540

Department of Mathematics, University of Kentucky, LeXington, Kentucky 40506 